\title{
Héritages républicains et résistances à "l'organisation impériale des savoirs »
}

Jean-Luc Chappey

\section{(2) OpenEdition}

1 Journals

Édition électronique

URL : https://journals.openedition.org/ahrf/7723

DOI : 10.4000/ahrf.7723

ISSN : 1952-403X

Éditeur :

Armand Colin, Société des études robespierristes

Édition imprimée

Date de publication : 1 décembre 2006

Pagination : $97-120$

ISSN : 0003-4436

Référence électronique

Jean-Luc Chappey, "Héritages républicains et résistances à « l'organisation impériale des savoirs » », Annales historiques de la Révolution française [En ligne], 346 | Octobre/Décembre 2006, mis en ligne le 01 décembre 2009, consulté le 22 avril 2022. URL : http://journals.openedition.org/ahrf/7723; DOI : https://doi.org/10.4000/ahrf.7723

Ce document a été généré automatiquement le 22 avril 2022

Tous droits réservés 


\title{
Héritages républicains et résistances à « l'organisation impériale des savoirs »
}

\author{
Jean-Luc Chappey
}

1 Que l'on se tourne vers le monde des sciences ou vers celui des lettres, les périodes consulaire et impériale marquent un profond bouleversement tant épistémologique et esthétique qu'institutionnel et social. Il est désormais établi que ce "moment 1800 » constitue une véritable rupture qui, selon la perspective historiographique choisie, est interprétée en terme de transition entre l'âge "classique » et l'âge "moderne », de passage des «Lumières» au «romantisme» ou de l'«encyclopédisme» au " positivisme ${ }^{1}$. S'écartant d'une histoire des idées trop peu soucieuse du contexte, de nombreux travaux ont cherché à inscrire ces transformations intellectuelles dans la dynamique des mutations politiques et sociales conduites par Napoléon Bonaparte entre 1799 et 1815 . Ces recherches ont déjà permis de renouveler en profondeur la connaissance des milieux scientifiques, littéraires ou philosophiques, et d'appréhender plus précisément l'analyse des rapports entre les dynamiques politiques et les logiques intellectuelles ${ }^{2}$. Parmi les nombreux chantiers qui restent encore ouverts sur ce terrain, la question de la portée politique et sociale de la construction des savoirs ayant l'homme pour objet reste encore largement posée. Dans mon travail sur la Société des Observateurs de l'homme (1799-1804) ${ }^{3}$, j'avais tenté de mettre en lumière les rapports entre l'émergence puis la disparition d'un projet scientifique (la construction d'une anthropologie) et les transformations radicales et rapides des espaces politiques et intellectuels qui rendent ce projet impossible et anachronique au début de l'Empire. Prenant appui sur ces résultats, je voudrais montrer que «l'échec» du projet des Observateurs s'inscrit dans un changement plus général d'organisation des savoirs qui renvoie et participe aux transformations majeures du régime impérial.

2 La mise en place de ce que je définis sous la notion «d'organisation impériale des savoirs » tend en effet à rendre impossibles certaines configurations de savoirs comme l'anthropologie des Observateurs, mais aussi la «science générale de l'homme» des 
Idéologues qui, incarnée au sein de l'Institut national ${ }^{4}$, a pu apparaître comme un véritable paradigme sous le Directoire. L'effacement progressif de ces projets face aux nouvelles normes institutionnelles et épistémologiques de construction des savoirs (caractérisées en partie par des logiques de polarisation, de professionnalisation et de spécialisation) constitue selon moi un élément fondamental du projet politique de Napoléon Bonaparte qui renvoie autant au rétablissement de l'esclavage qu'à la (re)constitution de nouvelles formes de hiérarchies et de dominations sociales, politiques ou sexuelles. On peut ainsi constater que cette remise en ordre de l'espace intellectuel (qui a permis à Napoléon Bonaparte de construire son image de "restaurateur" des sciences, des lettres et des arts) tend à faire disparaître une « organisation républicaine des savoirs» qui, sous des principes différents, pouvait réunir des projets autant scientifiques que politiques visant à «transformer » les hommes et les sociétés selon l'idéal de progrès et de régénération hérité des Lumières. Je voudrais montrer que, sous l'Empire, certaines oppositions et résistances ont pu se formaliser autour de la défense de cet héritage républicain, cette mission civilisatrice assignée aux « savants », débordant des frontières trop rigides des «Idéologues » ou de " groupe de Coppet ».

L'organisation impériale des savoirs

3 Sous le Directoire, l'Idéologie, cette "science des méthodes ", participe complètement à la construction du projet républicain et à la mise en place de l'idéal du citoyen "propriétaire ", comme l'illustrent le statut privilégié accordée à l'Institut national dans la Constitution de l'an III et la promotion politique et symbolique dont bénéficie la figure du savant, érigé en véritable "guide de la République ». Alors que de nombreux soupçons pèsent sur une raison dont on découvre les "maladies ", les sciences et leurs représentants sont appelés à travailler ensemble pour éviter la dégradation et le retour de la « barbarie » : ils doivent rendre de nouveau possible le progrès de la civilisation et fournir les instruments (l'analyse, l'observation...) susceptibles d'enfermer la raison dans de "justes bornes ", l'enthousiasme ou les passions étant désormais considérés comme des menaces à l'ordre politique et social ${ }^{5}$. À travers les différents mémoires qu'ils présentent devant leurs collègues de la Classe des sciences morales et politiques à partir de l'an IV, Pierre-Jean-Georges Cabanis et Antoine Destutt de Tracy posent les fondements théoriques, méthodologiques et institutionnels susceptibles de rendre possible une nouvelle mobilisation des savoirs et des savants en vue de garantir la stabilité de la République et de "terminer la Révolution $»^{6}$. Cette entreprise justifie la mise en place de contraintes importantes qui pèsent sur le monde des sciences, des lettres et des arts, contraintes qui s'incarnent dans l'organisation épistémologique et institutionnelle des savoirs, mise en place à partir de brumaire an IV autour de l'Institut national : rompant avec l'organisation académique et plus précisément avec les pratiques spécialisées et érudites, l'Idéologie (considérée ici au sens large) s'appuie sur une conception encyclopédique des sciences, et plus généralement des productions intellectuelles, qui légitime la nécessaire réunion de tous les savants, hommes de lettres et artistes autour d'outils et de normes communes. Cette " encyclopédie vivante $»^{7}$ doit permettre la construction d'une science générale de l'homme qui, fondée sur les principes sensualistes, servira de socle à la mission politique et sociale assignée aux savoirs et à leurs représentants : il s'agit en effet de transformer l'homme en changeant ses habitudes et ses sensations, perfectionner la société et les diverses populations qui la composent en s'engageant dans un processus de civilisation dont les savants doivent ouvrir la voie et garantir la bonne marche ${ }^{8}$. On le voit, l'Idéologie marque un 
élargissement considérable du terrain d'intervention de la science. Elle investit des terrains (les mœurs, la morale...) qui étaient auparavant contrôlés par d'autres institutions (l'Église). Elle s'affirme comme la nécessaire réunion des productions intellectuelles auparavant dispersées en différents domaines et pratiques de savoirs. Loin de se cantonner au statut d'organe central de l'espace intellectuel, l'Institut national s'impose comme un lieu essentiel dans les institutions politiques: ses membres, qui se réapproprient l'héritage des Lumières, doivent participer à l'entreprise de civilisation afin d'améliorer et de perfectionner les populations tant au niveau du «moral » que du " physique », mais aussi incarner une « opinion publique » susceptible de garantir l'équilibre entre les diverses institutions politiques et de préserver la nature républicaine du régime. En analysant les « rapports du physique et du moral ", en travaillant à la construction d'une grammaire générale et à l'étude des influences des climats, des institutions sur les populations, les savants doivent contribuer à la construction d'une nouvelle culture républicaine qui entend détruire toutes les formes de superstitions et de contestations ravalées au rang de "passions». Cette entreprise conduit indéniablement - du moins dans l'idéal - à l'institution du pouvoir des savants, "à leur ministère politique et à leur domination sociale ".

Alors qu'au lendemain immédiat de brumaire an VIII les Idéologues apparaissent clairement dans le camp des vainqueurs, l'ouverture des discussions sur le Concordat, les critiques véhémentes, et de plus en plus nombreuses, portées contre «l'empire des sciences ", marquent une transformation radicale par rapport au Directoire : dès 1800, le rôle accordé aux sciences et aux savants dans l'entreprise de civilisation et de régénération politique est mis en cause. Dans une lettre adressée à Joseph Droz, professeur de belles-lettres à l'École centrale du Doubs, Destutt de Tracy confie, en 1801, qu'il se trouve «dans le vide, du moins dans le moment des reculades $»^{10}$. En redonnant aux membres de l'Église leurs anciennes prérogatives, en favorisant la promotion de la figure du "poète » en opposition avec celle du savant, l'État joue un rôle essentiel dans la contestation des différents projets scientifiques fondés sur l'idéal de progrès et de régénération. Dès le Consulat, il apparaît que la science, ses outils et ses méthodes, ne sauraient conférer une suprématie à ses représentants et, de fait, légitimer leur intervention dans l'espace politique. Alors que les publicistes catholiques soutenus par des écrivains tels que Pierre-Simon Ballanche et René de Chateaubriand (et plus particulièrement les publicistes réunis du Mercure de France, fondé en janvier 1800 à l'initiative de Lucien Bonaparte) affirment, en valorisant le "sentiment ", que la nature humaine ne saurait se réduire à des lois physiologiques, le projet de construire une science générale de l'homme susceptible de servir de fondement au projet politique républicain perd de sa substance.

5 À bien des égards, l'année 1802 apparaît comme un tournant essentiel. La loi sur la réforme des institutions pédagogiques présentée par Fourcroy cette année-là, qui aboutit à renforcer l'autorité des professeurs des grandes institutions parisiennes, conjuguée aux difficultés qui traversent le monde de l'édition et à la politique de distribution des faveurs et des gratifications, ont pour conséquence de promouvoir une nouvelle «aristocratie» scientifique et littéraire. Ses membres tendent progressivement à s'arroger la qualité de "professionnels", qui instaurent comme dominantes et exclusives de nouvelles normes méthodologiques, et cherchent à confisquer un domaine de savoir dont les frontières correspondent à l'intitulé de leur chaire d'enseignement. Lorsque la même année, Cabanis tente de résister à cette évolution en publiant ses Rapports du physique et du moral, il est la cible, dans les 
journaux officiels, de violentes critiques qui l'accusent non seulement d'être "matérialiste ", mais surtout de vouloir faire dépendre la stabilité politique et sociale de la construction d'une science générale de l'homme et du travail commun d'une " caste » de savants; si ses adversaires ne remettent pas en cause l'utilité des sciences, ils dénoncent l'ambition de placer les sciences et les savants au cœur de l'entreprise politique. Le tournant anti-idéologique n'est donc pas à proprement parler un tournant anti-scientifique : l'entreprise qui est menée, à travers l'épuration du Tribunat, la réforme de l'Institut national et la suppression de la Classe de sciences morales et politiques en janvier 1803, vise surtout, d'une part, à rompre avec la mission régénératrice et civilisatrice conférée aux sciences et, d'autre part, à neutraliser, voire à supprimer, le pouvoir social et politique conféré aux savants, et plus généralement à la communauté savante incarnée encore par l'Institut national. Que les membres de l'Institut national soient exclus du Tribunat et que l'Institut national soit réduit à n'être qu'un espace de sociabilité intellectuelle me semblent illustrer cette volonté de distinguer nettement les sphères du pouvoir et du savoir et de réduire le champ d'intervention des savants aux domaines proprement scientifiques, une double stratégie qui s'inscrit complètement dans le processus de renforcement du pouvoir individuel de Bonaparte à partir de 1802 et dans la lutte menée contre les différents contre-pouvoirs. Si les projets sont fondamentalement différents dans leurs principes épistémologiques et méthodologiques, la disparition de la Société des Observateurs de l'homme en 1804 et de son projet encyclopédique s'inscrit dans la continuité de l'échec de la «science générale de l'homme » des Idéologues. L'idéal de "l'homme complet » $\mathrm{du}$ Directoire qui pouvait s'incarner dans certaines grandes figures du savant "philosophe » du XVIII siècle (Buffon ou Vicq d'Azyr) s'efface derrière la figure du savant «sédentaire » et du " professeur». Le processus de renforcement de l'autorité individuelle aux dépens du pouvoir collégial et communautaire caractérise autant l'espace politique qu'intellectuel.

6 Les effets de cette transformation sont multiples et bien connus : l'Institut national, comme l'a montré Roger $\mathrm{Hahn}^{11}$, perd sa position centrale dans l'organisation institutionnelle des savoirs, les sociétés savantes ou les journaux à vocation encyclopédique (la Revue philosophique, littéraire et politique ou le Magasin encyclopédique) tendent à disparaître à se spécialiser au profit de nouveaux espaces comme la fameuse Société d'Arcueil créée en $1808^{12}$. Sur le plan social et institutionnel, le régime encourage et favorise la (re)constitution d'une élite restreinte de "professeurs » des grandes institutions scientifiques et pédagogiques (Muséum d'histoire naturelle, École de médecine ou École polytechnique) qui tendent à s'arroger, par le contrôle exclusif des normes de validation scientifique, la promotion de nouvelles pratiques de connaissance et la confiscation des matériaux de savoir (les collections anatomiques ou botaniques, par exemple, mais pensons aussi aux enjeux qui entourent la mathématique algébrique) et ainsi à renforcer leur contrôle sur le domaine disciplinaire défini par leur chaire d'enseignement ${ }^{13}$. Sur le plan institutionnel, on assiste à un éclatement des différents lieux de savoirs, et au sein même de ces lieux de savoirs, Dorinda Outram l'a clairement montré pour le Muséum d'histoire naturelle ${ }^{14}$, à une polarisation des recherches justifiant les luttes que se livrent les différents professeurs. Les savants perdent leur indépendance politique ${ }^{15}$, mais certains gagnent en prestige et autorité ${ }^{16}$. En faisant éclater l'« encyclopédie vivante » auquel l'Institut devait donner corps, Bonaparte rompt avec l'idéal républicain et renforce son contrôle sur un espace savant dont chaque acteur tient désormais son autorité du Premier 
Consul et de l'empereur. Bonaparte en personne s'arroge désormais la place d'instance de validation et de consécration au sein de l'organisation intellectuelle comme le montre, dès le Consulat, son soutien à Volta ${ }^{17}$, la publication en 1808 des différents Rapports sur le progrès des sciences, lettres et arts et la création de l'Université.

Il semble possible de mettre en relation le processus de concentration de l'autorité politique entre les mains d'un seul et le processus de spécialisation et d'individualisation qui caractérise le monde scientifique et littéraire. Les systèmes de représentation sur lesquels est fondée l'identité du savant ou de l'écrivain se transforment: alors que, sous le Directoire, être savant, c'est marquer son appartenance à une communauté à travers la mention des appartenances multiples aux institutions de savoirs, l'Empire se caractérise par la promotion du "nom commun " suivi de la mention d'une appartenance unique; dans le monde des lettres, la valorisation de la figure du "génie" peut également être interprétée en terme d'individualisation de la figure de l'écrivain aux dépens de l'idée de «République des lettres ». L'autorité scientifique et littéraire tend ainsi à se concentrer entre les mains de nouveaux " patrons $»^{18}$, véritables homo clausus, qui, à l'instar d'un Georges Cuvier stigmatisent sous les notions d'«amateurs" ou de "charlatans» tous ceux qui s'opposent à ce processus général. Plus encore, en s'imposant comme l'instance de consécration intellectuelle et de contrôle des carrières scientifiques, littéraires ou artistiques, l'État provoque l'éclatement progressif de l'idéal d'une communauté intellectuelle homogène au profit d'une lutte d'intérêts individuels transformant certaines institutions en véritables champs de bataille. La promotion symbolique et les faveurs que reçoivent certains savants (pensons, par exemple, à Lacepède ou à Fourcroy) participent de cette même stratégie: la "notabilisation" des savants entraîne leur fusion au sein d'une élite plus large, au sein de laquelle la qualité même de "savant » tend progressivement à disparaître au profit de nouvelles pratiques de sociabilité mondaine et d'un nouveau système de distinction qui neutralise le pouvoir fondé spécifiquement sur la légitimité intellectuelle ${ }^{19}$. En octobre 1806, l'abbé Morellet ne pourra que constater avec regret l'affaiblissement symbolique du statut de « membre de l'Institut » dont l'habit ne suffit plus à ouvrir les portes de l'empereur :

«Voici encore un fait assez étrange qui peut être l'avant-coureur d'un état de choses fâcheux pour les Lettres en rendant plus difficiles et plus rares les relations des hommes de lettres avec le Gouvernement. Le 2e dimanche du mois de septembre 1806, Mr de Boufflers, membre de l'Institut dans le 2e classe et ci-devant l'un des Quarante de l'Académie française, venant d'entrer dans le salon de St. Cloud et se disposant à passer dans la galerie, un huissier lui a signifié qu'il ne pouvait entrer, que c'était par négligence de la part du Suisse, qu'il était parvenu jusqu'au salon, que sa consigne était expresse de ne laisse entrer aucune personne ayant le costume de l'Institut. Mr de Boufflers a eu beau représenter qu'il avait été toujours admis à faire sa cour à leurs Majestés dans cet habit, il n'a rien gagné ${ }^{20}$.

8 Les Rapports sur le progrès des sciences présentés à l'empereur en 1808 viennent légitimer les nouveaux découpages entre les différents domaines de savoirs et justifier les nouvelles positions de pouvoir. Par ses interventions, le régime consulaire et impérial contribue en effet à bouleverser les rapports de force au sein de l'espace intellectuel et à transformer l'ordre des savoirs aux dépens de la conception encyclopédique et communautaire défendue sous le Directoire. Que l'on se tourne vers l'histoire naturelle, les mathématiques, la chimie ou les statistiques départementales ${ }^{21}$, on constate (avec plus ou moins d'acuité) que la période impériale marque une rupture indéniable dans les modalités de construction du savoir, comme l'illustre la promotion scientifique et 
institutionnelle de l'anatomie comparée de Cuvier et des mathématiques de Laplace. On peut parler à cet égard d'un changement de paradigme et d'une transformation majeure des principes d'intelligibilité de l'homme. Ces mutations marquent également une rupture majeure dans le projet politique de l'Empire. S'interrogeant sur le rôle joué par les savants et les gens de lettres dans la légitimation de l'esclavage, que Bonaparte réinstaure en mai 1802, Yves Benot ${ }^{22}$ montre comment ce débat doit être inscrit dans une remise en cause générale du projet de civilisation et de la notion même de "progrès » et de "perfectibilité » humaine. Face à cette réorganisation consulaire et impériale des savoirs, l'historien semble être confronté à un mur du silence : celui, progressif et subi, des porte-parole des Idéologues, qui semblent baisser les armes à partir de 1804 et ne paraissent plus exprimer leurs désillusions et leurs mécontentements, timides et prudents, qu'à travers la correspondance entretenue avec Thomas Jefferson. Plus pesant encore, le silence complice, voire complaisant, des grandes sommités scientifiques (pensons à Jussieu, Lacepède, Monge, Berthollet ou Fourcroy) qui auraient renié leurs idéaux pour rallier opportunément un régime dont ils apparaissent comme les grands bénéficiaires. Les témoignages officiels sur l'état des sciences s'avèrent cependant souvent trompeurs. C'est par exemple le cas, si on le lit trop linéairement, du Rapport sur l'état des sciences de Cuvier : ce dernier s'érige dans ce texte en porte-parole d'un «noyau dur » de professeurs qui entendent renforcer leur position, légitimer davantage leur autorité et stigmatiser leurs adversaires. Loin d'être un « tableau » des positions de force, ce rapport, par ses « silences » ou, à l'inverse, les attaques particulièrement violentes qui sont portées, montre que, sous l'Empire, la position des «professeurs » et des membres de la nouvelle élite intellectuelle n'est pas encore totalement affermie. En somme, rien n'est encore joué et les différents textes publiés en 1808 sont des instruments destinés à exclure et marginaliser des résistances encore particulièrement vives contre l'organisation impériale des savoirs. Entre ceux qui s'opposent à l'abandon de la mission civilisatrice des savoirs, ceux qui tentent de résister à la dynamique de resserrement social de l'espace intellectuel ou d'éviter une marginalisation, la configuration des opposants est particulièrement complexe et ses représentants ne sauraient être réduits à l'étiquette trop réductrice d'« idéologues ». En fait, les oppositions qui prennent pour cible les partages des savoirs, les nouvelles formes d'autorité et s'attaquent directement à l'ordre politique et social incarné par Napoléon Bonaparte, se réunissent autour de deux idées : l'idéal de perfectionnement et l'organisation collégiale de la communauté intellectuelle.

9 Selon notre perspective, l'organisation impériale des savoirs permet de défendre la stabilité des groupes et des identités sociales: elle tend à graver dans le marbre les diverses distinctions et hiérarchies sociales et politiques établies d'autant plus fortement qu'elles peuvent alors se parer des oripeaux de la science ${ }^{23}$. Napoléon Bonaparte entend faire des sciences, d'une part des instruments d'une promotion personnelle et nationale, d'autre part des outils susceptibles de renforcer l'efficacité de l'administration ${ }^{24}$, une administration qui n'est plus considérée comme un moteur de la "régénération ", mais comme un outil de contrôle et de domination. Dans mon travail sur la Société des Observateurs de l'homme, traitant des «lectures impériales » qui s'imposent au sujet de l'enfant sauvage de l'Aveyron ou de l'expédition maritime du capitaine Baudin ${ }^{25}$, j'ai tenté de montrer comment les sciences ne sont plus considérées comme des instruments d'amélioration et de perfectionnement des hommes mais viennent légitimer le renfermement des individus ou des populations dans des catégories (le "sauvage", le «fou », mais cela est valable pour la femme, l'enfant, le 
pauvre...) sur lesquelles se greffent de nouvelles normes de contrôle et d'asservissement. En 1807, sont en effet publiés les résultats des deux grandes entreprises auxquelles ont participé les Observateurs : Jean-Marc-G. Itard publie son second rapport sur l'enfant sauvage de l'Aveyron ${ }^{26}$ et François Péron le récit du voyage aux terres australes ${ }^{27}$, deux ouvrages dont les frais d'édition sont pris en charge par les autorités. Les conclusions données révèlent le caractère désormais anachronique de l'idéal civilisateur dans lequel s'inscrivait le projet anthropologique des Observateurs. Le jeune enfant sauvage est déclaré "imbécile»dans le rapport présenté par le médecin Itard et est progressivement jugé incurable; les populations sauvages sont déclarées "inférieures" dans le récit de voyage de Péron, à la suite de mesures musculaires effectuées à l'aide du dynamomètre de Régnier. Ces deux exemples révèlent un phénomène plus général de construction d'une nouvelle sémiologie $d u$ corps social qui sert de fondement à la légitimation des nouvelles formes de domination mises en place à travers le rétablissement de l'esclavage ou le Code civil. Comme l'ont montré Marc Renneville dans son étude sur la criminologie ou Nicole Edelman sur l'hystérie féminine ${ }^{28}$, la science et ses nouveaux outils (la craniologie de Gall par exemple) jouent, sous l'Empire, un rôle essentiel dans la construction des lectures officielles sur l'infériorité naturelle des femmes et des sauvages ${ }^{29}$, sur la criminalisation des formes de déviance et de "folie», sur le statut des enfants et des pauvres. Ils légitiment les nouvelles segmentations du corps social. Dans tous les cas, la science "générale " de l'homme s'efface devant des approches thérapeutiques localisées: or «soigner" ne veut plus forcément dire "perfectionner». Sous l'Empire, l'hospice, comme la prison, deviennent moins des cadres de cure ou de réintégration possible que des espaces d'enfermement et de punition, évolution qui marquent une véritable rupture par rapport aux réformes des prisons et des hôpitaux entreprises pendant la période révolutionnaire. L'abandon de l'approche cosmologique en histoire naturelle, les critiques portées contre la "médecine philosophique», la marginalisation progressive de la "géographie descriptive " en faveur de la méthode algébrique, la réforme progressive des mathématiques dans l'enseignement dispensé à l'École polytechnique ${ }^{30}$, sont autant d'étapes qui rythment une transformation radicale dans la fonction assignée aux sciences: à défaut d'être des outils de perfectionnement des hommes et des sociétés, les différentes sciences et pratiques de savoirs ayant l'homme et les sociétés comme objets sont désormais appelées à devenir des outils de contrôle administratif, des instruments de reproduction des élites ou de légitimation du discours politique.

Est-ce aller trop loin que de tenter d'interpréter le succès obtenu par les théories «fixistes» de Georges Cuvier aux dépens du «transformisme» de Jean-Baptiste Lamarck à la lumière de cette question ? À partir de 1805, puis surtout en 1812 dans les Recherches sur les ossements fossiles de quadrupèdes, les thèses fixistes défendues par Georges Cuvier ne serviraient pas seulement à légitimer la théorie du déluge, mais viendraient légitimer davantage un nouveau paradigme, la fixité de l'ordre social et politique. Si Georges Cuvier peut être considéré comme la figure emblématique de ces transformations, c'est que l'anatomie comparée joue un rôle essentiel. Désormais, le caractère, le symptôme sont autant de signes renvoyant à des entités, collectives et individuelles, fixes et invariables, indépendantes et autonomes des "influences » extérieures. Dans ce processus, les sciences "officielles" jouent un rôle essentiel comme outils de validation et de justification à des discours de normalisation sociale et politique. Différentes normes issues des savoirs (la statistique, l'anatomie comparée) 
fournissent les outils nécessaires à fixer (après un temps de brouillage et de désordre lié à la Révolution) les identités sociales et collectives; dans cette perspective, il s'agit bien de «terminer » la Révolution au sens où sont rétablies des idées de distinction, de hiérarchie et d'ordre socio-politique ${ }^{31}$. La reconstitution de groupes séparés les uns des autres par des «fossés " infranchissables (dont les différences sont inscrites dans la nature) constitue un des fondements de cette organisation des savoirs sous l'Empire. On peut ainsi dire que l'Empire met progressivement en place une nouvelle représentation de la Nation, non plus organisée autour de la notion d'individu, mais de celle de groupes reliés les uns aux autres par la figure de l'Empereur.

La défense de l'idéal du progrès

11 Si l'on peut parler d'héritages républicains dans la formalisation des résistances à cette évolution, celle-ci se traduit dans l'opposition à cette volonté de fixer les identités individuelles et génériques et de nier l'idée de perfectionnement des hommes et des sociétés. Dans cette perspective, la défense d'une "science générale de l'homme" constitue un enjeu essentiel. En 1807, dans ses Lettres philosophiques, Rigomer Bazin en appelle à une "révolution morale » rendue possible par la connaissance de l'homme. Face à ceux qui veulent "arrêter» le mouvement de la liberté, il affirme que le " progrès des sciences » est encore nécessaire pour transformer l'homme et régénérer la sociétée ${ }^{32}$. Pour ceux qui défendent cet idéal républicain, ce progrès ne saurait être possible dans le contexte de spécialisation et de partage des savoirs. Ils défendent au contraire la nécessaire unité des savoirs : c'est le cas des « héritiers de Buffon » qui se regroupent autour du naturaliste Sonnini de Manoncourt ${ }^{33}$, mais aussi d'un Moreau de la Sarthe qui s'érige, dans les colonnes de la Décade puis de la Revue philosophique, en défenseur de la "médecine philosophique" et, plus précisément encore, du «traitement moral » de Pinel ${ }^{34}$. Bien qu'ils puissent être opposés sur certains principes, ils se rejoignent, sur le plan théorique, autour d'autres: la défense de la thèse des «rapports du physique et du moral», qui sert de support à l'idée selon laquelle la nature de l'homme et sa condition individuelle et collective peuvent être améliorées par une transformation de son «environnement» et de son «milieu». Cette prise de position justifie encore la mission assignée aux savoirs et à leurs représentants dans le progrès social et politique et légitime leur intervention dans des domaines aussi variés que la morale ou l'éducation. On comprend qu'ils fustigent le « renfermement » de la science et des savoirs dans des "boîtes noires ", une position qui passe, par exemple, par la défense d'une conception littéraire et philosophique de l'écriture scientifique ${ }^{35}$. Ces résistances se formalisent particulièrement à travers la défense d'un " héritage " théorique et la valorisation d'auteurs qui s'appuient sur des stratégies de rééditions qui ne sauraient être réduites à des enjeux commerciaux : l'entreprise de réédition des œuvres de Buffon menée par les naturalistes réunis autour de Sonnini de Manoncourt, celle des ouvrages des "médecins philosophes » (Vicq d'Azyr et Pinel) réalisée par Moreau de la Sarthe témoignent de cette volonté de construire une sorte de Panthéon venant concurrencer les représentants de la nouvelle «aristocratie » intellectuelle choisis par le régime et de proposer un contre-modèle théorique à l'ordre impérial des savoirs.

12 Dans tous les cas, il s'agit de s'opposer aux partisans de la spécialisation des savoirs accusés de vouloir empêcher la possibilité de progrès. L'opposition virulente de Sonnini de Manoncourt à Cuvier et aux "anatomistes », comme celle de Moreau de la Sarthe aux "physiologistes", témoigne de la lutte menée contre la «localisation" des approches et la fragmentation des savoirs sur l'homme et les sociétés, une évolution qui 
tend à marginaliser l'approche cosmologique et civilisatrice et à légitimer l'idée que la nature de l'homme est fixe et intangible. C'est une des raisons des attaques lancées par Moreau de la Sarthe dans les colonnes de la Décade, en 1804-1805, contre les thèses de Gall, au moment où se dernier bénéficie du soutien du régime. Selon Moreau, la possibilité de réduire la connaissance de la nature humaine à celle du cerveau constitue non seulement une ineptie sur la plan scientifique, mais surtout un véritable danger pour l'ordre politique et social; la « localisation » des actions humaines pose en effet le problème de la « responsabilité » individuelle et a des conséquences particulièrement dangereuses dans la pratique judiciaire : l'émergence d'une expertise médicale fondée sur la phrénologie aurait en effet pour conséquence d'établir une " nature » criminelle (qu'il suffirait de reconnaître) et donc de transformer la justice en une pratique punitive et non plus éducative. Refusant de réduire la science de l'homme à un domaine de connaissance (l'anatomie comparée) ou à un objet particulier (comme le cerveau), Sonnini de Manoncourt et Moreau de la Sarthe défendent cet idéal de progrès que l'on peut définir comme républicain. À travers le regard «anthropologique » qu'ils portent sur les groupes sociaux, sexuels et sur certaines populations lointaines et isolées ${ }^{36}$, ils continuent de se retrouver dans un projet qui recouvre tous les domaines de la vie en société (de l'économie à la politique en passant par la culture et l'enseignement) et s'étend également à toutes les populations : s'il s'agit en effet de perfectionner la société française, il s'agit aussi de resserrer les liens de la «famille universelle » et de faire accéder tous les peuples d'Europe, du globe, au bonheur.

Sonnini de Manoncourt et Moreau de la Sarthe ne sont pas isolés : ils appartiennent à une configuration plus large réunissant à partir des années 1802-1803 des acteurs qui, souvent victimes des transformations de l'organisation institutionnelle et sociale de l'espace intellectuel, prennent position contre les fondements théoriques de l'ordre impérial des savoirs. Parmi eux, on peut citer certains médecins de l'École de Montpellier (tel que Jean-Baptiste-T. Baumes), mais aussi des partisans du galvanisme (René Tourlet, Jean Nauche) ou de la géographie descriptive (Alexandre de Ferrières ou Louis-Joseph-Philippe Ballois). Entre ces différents acteurs et courants théoriques s'établissent des relations par le biais de jeux de réseaux et d'actions, qui s'organisent souvent autour de personnalités intellectuelles de premier plan: on peut constater ainsi le rôle joué par Lacepède, Parmentier, Chaptal ${ }^{37}$ dans cette nébuleuse de résistances. Ce constat contraste avec le «silence» et la passivité dont sont souvent accusées ces grandes figures du monde intellectuel. Car, si elles affectent de se conformer aux règles officielles pour se bâtir une position dominante au sein de la nouvelle élite impériale, ces personnalités scientifiques savent également utiliser des réseaux constitués de personnalités moins en vue pour intervenir et sans doute tenter de s'opposer à certaines évolutions qui affectent l'ordre des savoirs. Elles dénoncent entre autres le nouveau partage des compétences entre les lettres et les sciences, un partage fondé sur la conception d'un dualisme irréductible entre l'âme et le corps rendu officiel à travers la valorisation de la littérature considérée comme l'expression des sentiments et l'émergence de la figure du "poète"; elles refusent par là la réduction des terrains d'intervention des "savants" aux seuls objets physiques ou physiologiques aux dépens des terrains qu'ils avaient investis, comme celui de la morale. On peut ainsi mesurer l'extrême complexité théorique et sociale de ces résistances qui ne sauraient se réduire à un groupe ou une école de pensée (les «Idéologues»). Très hétérogènes, elles révèlent au contraire des alliances d'intérêt souvent contre-nature et des constructions théoriques suffisamment originales pour 
provoquer des attaques particulièrement violentes. On pourrait presque parler d'une stratégie de l'«aberration ", qui permet de comprendre la place et le rôle de théories ou de pratiques apparaissant fantaisistes, et servant en réalité de supports aux différentes résistances.

S'interroger en effet sur les résistances intellectuelles sous l'Empire nécessite de dépasser ce mur des silences pour se tourner vers des acteurs et des théories présentés dans l'historiographie comme "mineurs", " anachroniques" voire "aberrants». Ce sont justement les enjeux qui entourent leur marginalisation au sein de l'espace intellectuel qu'il convient d'interroger. Présentées trop souvent dans l'histoire des sciences comme des "anachronismes", des "aberrations", donc des "erreurs", les appropriations d'héritages et bricolages théoriques doivent être pris au sérieux et considérés comme des instruments de l'offensive menée contre la mise en place des nouvelles normes méthodologiques et épistémologiques. Lorsque Moreau de la Sarthe réédite, en 1806, la physiognomonie de Lavater, il s'agit pour lui de promouvoir une théorie lui permettant de défendre le principe des rapports du moral et du physique et l'idée d'une possible transformation de la nature humaine. Si cette réédition lui vaut d'être violemment attaqué par les représentants des sciences, il place en fait son intervention sur un terrain plus général, qui est celui de l'ordre politique et social : la théorie de Lavater lui sert en effet encore une fois à lutter contre toutes les nouvelles formes de fragmentation du savoir sur l'homme. Dans cette perspective, il est possible de mieux mesurer la portée de certains textes qui sont trop souvent étudiés de manière isolée : pensons aux Quelques mémoires ${ }^{38}$ de Samuel Dupont de Nemours en 1807. Parmi ces textes censés compléter la Philosophie de l'Univers, le mémoire intitulé Sur l'Instinct va être l'objet d'une vive polémique avec Georges Cuvier ${ }^{39}$. Dupont de Nemours pose comme principe l'unité du règne naturel et postule la validité des rapports entre le physique et le moral: selon lui, les mœurs et les rapports sociaux constituent les «influences» majeures de la transformation de l'organisation animale et humaine. Pour Laurent Loty, il infléchit ainsi les thèses proposées par Cabanis et s'écarte des discours officiels qui souhaitent voir distinguer nettement ce qui relève de l'organisation physique (anatomie et physiologie) de ce qui relève de la morale (littérature et religion) ${ }^{40}$. Ce qui est intéressant ici, c'est que Dupont de Nemours que l'on ne classerait pas parmi les « opposants républicains » sous l'Empire, s'inscrit, par ce texte, dans la configuration des résistances à l'ordre impérial des savoirs.

Il est possible de dire que c'est à travers la réunion de ces diverses résistances très hétérogènes que se construit sous l'Empire cet "héritage des Lumières" (parallèlement à «l'héritage de Buffon ») qui se formalise, dans le contexte particulier de la période, autour de la défense de l'idée de progrès et de civilisation. La publication en 1809 de la Philosophie zoologique de Lamarck est sur ce point particulièrement importante : on peut en effet faire l'hypothèse qu'elle sert de pôle de cristallisation de ces différentes formes de résistances. Il semble en effet, à la lumière des débats qu'elle provoque, que la théorie du "transformisme» est alors considérée comme un outil efficace pour lutter contre les nouvelles formes de normalisation politique et sociale. Ce qui justifie en large partie la violence avec laquelle Lamarck, pourtant professeur au Muséum d'histoire naturelle, est combattu. La critique porte autant sur la théorie qu'il défend que sur le fait que le transformisme devient le point de ralliement de tous les exclus des institutions dominantes. Ils voient dans la défense et la promotion de cette théorie un moyen de mettre en cause l'autorité des savants officiels. On retrouve parmi les diffuseurs et promoteurs du transformisme les opposants déjà cités à l'organisation 
impériale des savoirs tels que Sonnini de Manoncourt ou Tourlet. Par le biais des journaux en particulier, ils cherchent à diffuser la théorie de Lamarck auprès d'un large public et tentent ainsi d'instaurer ce dernier en instance de validité de la vérité scientifique. On comprend dès lors pourquoi l'État cherche à intervenir directement, en 1809, pour imposer la théorie du catastrophisme défendue par Cuvier. Il s'agit moins de réduire cette intervention à la défense de la religion catholique - même si cette dimension ne peut être occultée - que de l'interpréter comme une stratégie visant à défendre son contrôle exclusif des normes de validité du savoir scientifique et sa position d'instance de la vérité scientifique.

16 L'originalité de ces résistances est en effet qu'elles s'appuient en très large partie sur des stratégies éditoriales particulièrement efficaces. Soutenus par des personnalités établies au sein même du système intellectuel et administratif (Chaptal, Lacepède ou Parmentier), les acteurs de cette opposition théorique à l'ordre impérial des savoirs, sont surtout bien implantés au sein du monde de la librairie et de l'imprimerie parisienne, particulièrement au sein de la presse. On connait la victoire commerciale, et scientifique, remportée par le Dictionnaire d'histoire naturelle dirigé par Sonnini de Manoncourt et l'imprimeur Déterville sur celui de Cuvier et l'imprimeur Levrault ${ }^{41}$. Plus largement, la presse joue un rôle essentiel dans l'organisation de cette résistance : le Journal de Physique de Jean-Claude Delamétherie devient dès 1802 le fer de lance de la lutte contre les "nouveaux professeurs"; autour de la Bibliothèque Physico-économique de Sonnini ${ }^{42}$, de la Décade philosophique, dont Moreau de la Sarthe est un des rédacteurs les plus actifs, se dessinent des réseaux et se créent des solidarités. Le fait que la presse constitue un terrain privilégié de cette opposition ne s'explique pas uniquement par des raisons d'opportunisme : la relation entretenue avec un large public (on retrouve ici la question de la "vulgarisation $»^{43}$ ) et la volonté de défendre la conception d'une organisation collégiale et "ouverte" des savoirs justifient le rôle majeur joué par l'économie éditoriale dans la formalisation de ces résistances. À travers leur position de «publicistes» scientifiques et leur combat en faveur d'une science qui doit rester accessible au « large » public (justifiant ainsi la défense d'une écriture « littéraire » de ses observations et résultats), les auteurs défendent le fonctionnement collégial, communautaire et indépendant de la communauté intellectuelle face au renforcement du pouvoir de Napoléon Bonaparte.

La question de la communauté intellectuelle

17 En favorisant la polarisation des différents domaines et pratiques intellectuels, en mettant en cause la figure du savant construite sous le Directoire, le nouveau régime consulaire entend se prémunir contre une communauté savante susceptible d'incarner l'opinion publique éclairée et d'utiliser son autorité intellectuelle pour intervenir dans l'espace politique. Les mesures prises entre 1807 et 1808 contre certains journaux, en particulier la Décade philosophique ou les Archives littéraires de l'Europe ${ }^{44}$, me paraissent s'inscrire dans cette volonté du régime de circonscrire les espaces de discussion scientifique situés en dehors des grandes institutions savantes, d'éviter que se constitue ce que Destutt de Tracy définit, dans son Commentaire sur l'Esprit des lois de Montesquieu, comme une "démocratie de la raison éclairée ». Le conflit opposant Georges Cuvier à ses adversaires (Gall, Lamarck en particulier dans les années 1804-1809) prouve que la place assignée au public est l'un des enjeux essentiels des luttes pour un ordre impérial des savoirs. En 1807-1808, la querelle entre Gall et Cuvier porte sur l'approche " encyclopédique » des sciences, Cuvier attaquant le caractère "philosophique » des travaux de Gall et lui reprochant de «tirer» de ses analyses anatomiques des leçons 
portant sur le moral alors que tout ce qui ressort du «moral » devrait être désormais exclu des travaux proprement scientifiques. Cuvier rejette ainsi les travaux du phrénologiste du côté de la pseudo-science. Comme l'a montré Dorinda Outram, le grief principal que Cuvier porte contre Gall est sa relation au public. C'est parce qu'il aurait voulu bâtir sa renommée auprès d'un large public que Gall ne pourrait être considéré comme un «vrai » savant : "Gall vint à Paris ; il présenta à l'Académie ses découvertes sur le cerveau, témoigne Cuvier. Mais il réserva sa craniologie pour le vulgaire ; mon rapport sur son mémoire a été un assez grand travail ; malgré la manière honorable dont je parlai de lui, il crut, à la manière des charlatans, devoir répondre un gros volume à quelques restrictions que j'apportais à ses propositions. Il espérait que je répliquerais et que nous occuperions ainsi le public, mais je me gardai bien de me donner dans le piège $»^{45}$. Comme Cuvier l'écrit encore à son ami de Tübingen, KarlFriedrich Keilmayer, en 1808 : «Dans ce pays-ci, la première règle de conduite pour un savant c'est de ne jamais parler de ce qui est livré au grand public et aux journaux; en un mot des objets de la curiosité populaire. Ce serait se traîner dans la boue et se livrer aux bêtes $»^{46}$. Fi de l'idéal de la "communauté intellectuelle » et du rôle d'arbitre assigné au public dans les débats: il faut désormais neutraliser le pouvoir d'une "République des savants", réduire le public au rang de simple spectateur face au monde politique et intellectuel.

Si l'on me permet cette hypothèse, l'adoption en 1810 du Code de la Librairie, qui remet en place, par l'intermédiaire des brevets, un contrôle administratif sur le monde de l'édition, ne pourrait-il être interprété dans cette perspective? Ce Code est moins le produit d'une volonté autoritaire qu'une réponse aux attentes des imprimeurs et libraires les plus importants qui, face à la crise qui frappe le secteur de l'imprimé depuis 1802, se tournent vers l'État afin de mettre fin à ce qu'ils définissent comme une véritable anarchie commerciale ${ }^{47}$. Sous l'Empire, leurs revendications, et les attaques qu'ils portent sur les "petits » imprimeurs et libraires, sont relayées par les acteurs dominants de l'espace scientifique et littéraire, ces «professeurs » qui accusent leurs adversaires de profiter des « abus » et des dysfonctionnements de l'économie éditoriale pour s'approprier, de manière complètement illégitime selon eux, le statut de savant et d'homme de lettres. Le monde de l'édition est ainsi traversé par les luttes qui agitent l'espace intellectuel et, à l'inverse, ces luttes se chargent d'enjeux éditoriaux, d'autant plus que, dans les faits, les entreprises éditoriales et commerciales lancées par les "professeurs" sont celles qui se heurtent aux échecs les plus retentissants. Sous l'Empire, ceux qui revendiquent le statut de "professionnels » n'ont pas encore gagné et le public de «spécialistes » auquel ils prétendent s'adresser reste encore largement à construire. Seule une étude plus précise des modalités de distribution des «secours et pensions $»^{48}$ permettrait de préciser davantage le phénomène mais il apparaît d'ores et déjà que l'État cherche à reconstituer une élite restreinte et choisie, cumulant les fonctions institutionnelles les plus élevées dans les institutions intellectuelles avec les gratifications et les postes honorifiques les plus prestigieux.

19 Face à cette normalisation du «milieu » des savants et des écrivains, les initiatives éditoriales se multiplient pour contester la fermeture des carrières, le contrôle de l'État et sa volonté d'exclure de l'espace intellectuel. C'est dans cette perspective que, dans le monde des lettres plus particulièrement, il est possible de comprendre l'essor quantitatif des dictionnaires qui, sous des titres très différents, proposent des listes de noms d'auteurs «contemporains $»^{49}$. Dans tous les cas, il s'agit de démontrer que les contours du monde littéraire sont beaucoup plus larges que ceux érigés par les 
autorités et les écrivains consacrés, de défendre le fait que l'identité d'écrivain ne saurait être confisquée par une élite restreinte et choisie par l'empereur. Ces publications, qui concernent entre autres les domaines de la médecine ou de l'histoire naturelle, tendent à valoriser le «beau style » et à promouvoir la figure du «savantphilosophe ou écrivain » aux dépens de la distinction entre les sciences et les lettres, en appellent au "public», instance de légitimité et de reconnaissance. En intervenant directement dans le monde de la librairie et de l'imprimerie et en établissant des contraintes administratives qui brident les règles de l'économie éditoriale, l'État répond au contraire aux attentes des « dominants ", qu'ils soient membres de l'espace éditorial ou intellectuel, réduisant le public (selon la même logique que l'on constate sur le terrain politique) au rang de spectateur. En adoptant le Code de la Librairie, l'État, une nouvelle fois, intervient pour renforcer la spécialisation, la polarisation et la hiérarchisation du monde des sciences et des lettres et pour accélérer la marginalisation de l'idéal communautaire, encyclopédique et civilisateur.

20 À partir de 1810 cependant, à la suite de la loi sur l'imprimerie et la librairie, on peut constater la multiplication des initiatives en faveur de la reconstitution de sociétés privées. Elles se veulent réunions d'actionnaires, susceptibles - à l'instar des sociétés savantes créées en 1790, comme le Cercle social ou la Société nationale des neuf sœurs d'encourager la publication d'ouvrages en dehors du contrôle administratif de l'État, à l'écart des contraintes commerciales. Le danger de telles entreprises est pointé du doigt dans un rapport établi en 1813 par la Direction générale de l'Imprimerie :

« Un vieil idéologue, M. [Marc-Ferdinand] Groubert de Groubentall qui avait publié il $\mathrm{y}$ a vingt ans quelques rêves politiques et philosophiques, après avoir dormi pendant les orages de la révolution [... ] veut nous enrichir d'un mémoire sur les moyens d'améliorer le sort des gens de lettres. Si ces projets à ce sujet n'étaient pas de la folie, on pourrait le laisser déraisonner à son aise, mais ses folies ont du danger et il est convenable de le prévenir. Son idée est qu'il n'y a de bon que la littérature et les gens de lettres; qu'un état ne peut bien aller que par eux, parce qu'ils sont la source de toute la lumière. Il crée en conséquence un empire littéraire gouverné par l'aristocratie de l'Institut. Après en avoir dit tout le mal qu'il a pu imaginer, il abolit la censure que le gouvernement a considéré comme une sauvegarde nécessaire. Il défend la mise en vente de tous les ouvrages à moins d'en avoir obtenu le privilège de l'empire littéraire qui ne l'accorde qu'à un prix d'enchère et seulement pour dix ans [...]. L'Empire littéraire laisse jouir les auteurs vivants de la propriété de leurs ouvrages, mais elle passe à leur mort à l'Empire [...]. L'idée de faire une corporation des écrivains, si elle pouvait être suivie, produirait une institution bien autrement formidable que n'ont pu l'être la Sorbonne, les Jésuites, les moines, les sectes secrètes et même l'inquisition. [...] Rien ne manque à ce plan de ce qui pourrait donner aux écrivains une fortune et une autorité tout à fait indépendante du gouvernement [...]. $»^{50}$.

21 Affubler Groubert de Groubentall de La Linière de l'étiquette d'«idéologue " est particulièrement révélateur de l'interprétation du danger. Le problème qui se pose ici justifie la réaction défensive des autorités contre des lieux au sein desquels peut se construire, en dehors de leur contrôle, un jugement et se diffuser une réputation littéraire, scientifique, voire esthétique ${ }^{51}$. Force est de constater qu'à partir de 1810 , c'est du monde des lettres que provient cette résistance, résultat paradoxal s'il en est, $\mathrm{du}$ processus, encouragé par Napoléon Bonaparte dès son arrivée au pouvoir, de promotion de "l'homme de lettres » contre le savant : la promotion s'avère tellement couronnée de succès qu'en conséquence se construit une nouvelle figure de l'écrivain, insoumis aux autorités et défendant, au nom du "génie », une nécessaire autonomie. 
C'est, en partie, l'enjeu des débats sur les prix décennaux qui opposent l'empereur aux représentants éminents du monde des lettres ${ }^{52}$. En cherchant à diminuer le " pouvoir " de l'Institut national, et plus généralement, en favorisant l'éclatement de la communauté intellectuelle et en rendant anachronique l'idéal encyclopédique, Napoléon Bonaparte cherche non seulement à neutraliser un possible contre-pouvoir (incarné à travers l'idée d'une "République des savants »), mais aussi à marginaliser cette mission de civilisation assignée aux savants et gens de lettres. On assiste, à la fin de l'Empire, à un renversement intéressant : alors que, sous le Directoire, cette mission reposait particulièrement sur les représentants des sciences (physiologistes et naturalistes particulièrement), on peut constater que cette idée est revendiquée à partir de 1810 par les écrivains dans la lutte qu'ils mènent contre le régime. Si cette opposition des hommes de lettres sous l'Empire ne saurait être considérée comme "républicaine " (certains défendent des positions complètement opposées ${ }^{53}$ ), elle permet de nuancer certaines interprétations concernant la figure du "poète" caractéristique de cette période ${ }^{54}$. Loin de se retrancher dans les limbes de «l'art pour l'art », le « poète » revendique l'idée d'utilité et de mission qui le place, il me semble, dans un héritage "républicain »: si les principes défendus sont différents - voire antinomiques -, le savant du Directoire et le " poète » de la Restauration ${ }^{55}$ défendent tous les deux l'idée selon laquelle les productions scientifiques ou littéraires peuvent transformer l'individu et les sociétés et contribuer à améliorer le bonheur commun, idée qui sera reprise sous différentes formes pendant tout le XIX ${ }^{e}$ siècle et qui peut être considérée comme un legs essentiel de la période révolutionnaire : on pourrait ainsi comprendre les tensions qui émergent dès l'Empire et ne cessent de s'exacerber sous la Restauration entre les écrivains " romantiques ", les autorités et surtout l'Église ${ }^{56}$.

Parfois particulièrement violentes, les luttes intellectuelles qui caractérisent la période impériale n'ont pas uniquement comme enjeux le contrôle des positions institutionnelles, des matériaux de savoirs ou des clientèles. Elles renvoient à des oppositions profondes touchant autant aux rapports entre l'État, les savants et les écrivains, qu'aux fonctions assignées aux sciences et aux lettres dans l'organisation sociale et politique. L'Empire est, sur ce terrain également, une période particulièrement intéressante pour étudier la confrontation entre différentes conceptions et modèles d'organisation d'un espace intellectuel dont les dynamiques doivent être intégrées dans les transformations plus générales qui touchent les formes de pouvoir et de domination. Au risque de simplifier et de réifier certains phénomènes, on aurait tort de vouloir réduire les "héritages républicains" à des groupes, des milieux ou des systèmes théoriques homogènes. Appréhendés dans le contexte précis des conflits et des oppositions qui traversent le monde des sciences et des lettres entre 1804 et 1815 , ces héritages républicains apparaissent souvent comme des "bricolages » réalisés par des acteurs cherchant à s'opposer à une organisation dominante et officielle qui ne cesse à son tour de se transformer. Plutôt que de raisonner en termes de conflit manichéen, cette constatation doit nous obliger à repenser dialectiquement les oppositions qui traversent l'espace intellectuel, ce qui permettrait de mieux comprendre la nature de ce régime impérial qui hésite entre république et monarchie. $\mathrm{Si}$ les bouleversements majeurs qui caractérisent l'ordre institutionnel et épistémologique des savoirs renvoient indéniablement à une marginalisation progressive du projet républicain du Directoire, l'Empire voit également émerger des lieux de conservation où ce projet prend des formes novatrices et originales et continue ainsi d'être défendu. Ce processus permet d'expliquer que, dès la chute de l'Empire, cet 
héritage républicain est encore réinvesti, non seulement par le biais de programmes théoriques ${ }^{57}$, mais aussi à travers de nouvelles entreprises intellectuelles qui, loin de cultiver la nostalgie, cherchent à redonner corps et à l'idéal républicain et au projet de civilisation. C'est par exemple le cas de la Revue encyclopédique (1819-1833) de Jullien de Paris qui défend, dans un contexte de forte spécialisation des périodiques scientifiques et littéraire, la conception d'un lieu de publication encyclopédique susceptible de servir de support à une mission civilisatrice assignée aux sciences et aux lettres ${ }^{58}$. Il serait intéressant de suivre les prises de position successives en faveur de l'idéal encyclopédique et communautaire ${ }^{59}$ et de relier les entreprises intellectuelles qui s'y reportent aux projets politiques du XIX ${ }^{e}$ siècle. Dans cette perspective, il conviendrait sans doute de s'interroger davantage sur les enjeux de la "redécouverte» historiographique des milieux et des projets intellectuels du Directoire dans les années 1880-1890. On pourrait ainsi mieux saisir les formes de réappropriation de cet héritage républicain du Directoire et du Consulat dans la construction du projet politique de la Troisième République (pensons plus précisément au projet pédagogique, mais aussi colonisateur) et dans l'émergence de la figure de l'intellectuel à la fin du XIX ${ }^{e}$ siècle $^{60}$, une autre voie possible pour mesurer davantage le rôle de la Révolution dans la construction de «l'idée » et des pratiques républicaines ${ }^{61}$.

\section{NOTES}

1.Pour une mise au point récente, voir Claude BlANCKAERT et Michel PORRET (dir.), L'Encyclopédie méthodique (1782-1832). Des Lumières au positivisme, Genève, Droz, 2006. 2.Parmi les travaux récents, signalons Bruno BELHOSTE, La formation d'une technocratie. L'École polytechnique et ses élèves de la Révolution au Second Empire, Paris, Belin, 2003 ; Charles C. GILLISPIE, Science and Polity in France: The Revolutionary and Napoleonic Years, Princeton and Oxford, Princeton University Press, 2004 ; Patrice BRET, « Nouvelles lectures de l'histoire des sciences et des techniques sous la Révolution ", dans JeanClément MARTin (dir.), La Révolution à l'œuvre. Perspectives actuelles dans l'histoire de la Révolution française, Rennes, PUR, 2005, p. 317-331; Dominique MARGAIRAZ, François de Neufchâteau. Biographie intellectuelle, Paris, Publications de la Sorbonne, 2006.

3.Jean-Luc CHAPPEY, La Société des observateurs de l'homme (1799-1804). Des anthropologues au temps de Bonaparte, Paris, Société des études robespierristes, 2002.

4.Josianne BoULAD-AYOUB et Martin NADEAU, « La Décade philosophique » comme système 1794-1807, Rennes, PUR, 2003.

5.François Azouvi (dir.), L'institution de la Raison. La révolution culturelle des idéologues, Paris, Vrin, 1992, avant-propos.

6.Comme le note Brigitte SCHLIESSEN-LANGE, « il s'agissait d'ériger un système philosophique contre la menace de perte et recul des Lumières ", Idéologie, Révolution et uniformité de la langue, Paris, Mardaga, 1996, p. 193.

7.Jean-Luc CHAPPEY, « Usages et enjeux politiques d'une métaphorisation de l'espace savant en Révolution : “l'Encyclopédie vivante” de la République thermidorienne à 
l'Empire », Politix, «Les savants et le politique », 48, 1999, p. 37-70 ; « Raison et citoyenneté : les fondements culturels d'une distinction sociale et politique sous le Directoire ", Citoyen et citoyenneté sous la Révolution française, Actes du Colloque de Vizille du 24-25 septembre 2005, Paris, Société des études robespierristes, 2006, p. 279-288. 8.Xavier MARTIN, « Révolution française et socialisation de l'individu », dans $L a$ Révolution française et les processus de socialisation de l'homme moderne, IRED / Université de Rouen, Éditions Messidor, 1985, p. 77-84.

9.Dominique DAMMAME, « Entre science et politique, la première science sociale », Politix, 1995, 29, p. 29 ; du même, Histoire des sciences morales et politiques et leur enseignement des Lumières au Scientisme, thèse dactylographiée, Université Paris ISorbonne, 1982 ; Martin S. STAUM, « L'image de la Révolution française dans la classe des sciences morales et politiques ", dans Michel Vovelle (dir.), L'image de la Révolution française, Pergamon Press, 1990, vol. I, p. 530-534.

10.AN, $474 \mathrm{Mi} / 1$, Archives privées de Joseph Chappey, lettre de Destutt de Tracy du 25 brumaire an X (16 novembre 1801).

11. Roger HAHN, L'anatomie d'une institution scientifique. L'Académie des sciences de Paris, 1666-1803, Paris, Éditions des Archives contemporaines, 1993, p. 445.

12.Nicole et Jean DHOMBRES, Naissance d'un nouveau pouvoir : sciences et savants en France, 1793-1824, Paris, Payot, 1989 ; Robert Fox, « Scientific Enterprise and Patronage of Research in France, 1800-1870 », Minerva, London, 1973, 11, p. 445-449 ; Maurice CROSLAND, The Society of Arcueil. A View of French Science at the Time of Napoleon I, London, Heinemann, 1967, p. 4-55.

13.La polarisation des lieux de recherches et la formalisation des domaines disciplinaires ne touchent pas seulement les sciences : à partir de l'Empire, la philosophie redevient ainsi une « affaire de professeur » et l'on assiste à une « institutionnalisation » de la philosophie qui acquiert ainsi des « lieux propres ». Cf. Dinah RIBARD, Raconter, Vivre, Penser. Histoires de philosophes 1650-1766, Paris, Vrin, 2003, p. 39 et suiv.

14.Dorinda OUTRAM, « Le Muséum national d'histoire naturelle après 1793 : institutions scientifique ou champ de bataille pour les familles et les groupes d'influence ", dans Claude BLANCKAERT et alii (dir.), Le Muséum au premier siècle de son histoire, Paris, Éditions du Muséum national d'histoire naturelle, 1997, p. 25-30. Voir aussi Emma C. SPARY, Utopia's Garden. French Natural History from Old Regime to Revolution, The University of Chicago Press, 2000.

15. « À travers ses efforts généreux pour soutenir la science, Napoléon mit fermement en place la subordination de la société savante à l'État. Il soutenait la science, mais en retour il lui demandait une loyauté absolue envers la nation et ses opinions politiques ». Roger HAHN, L'anatomie d'une institution scientifique, op. cit., p. 443. 16. Maurice CROSLAND, Science under Control. The French Academy of Science, 1795-1914, Cambridge, Cambridge Univ. Press, 1992, p. 301-302.

17.Pancaldi GIULIANO, Volta, science and culture in the age of the Enlightenment, Princeton, Princeton University Press, 2003. Voir aussi Stéphanie DeProuw, Un héritage des Bonaparte : le prix Volta (1802-1815 ; 1852-1888). Une tentative d'encouragement à la recherche en électricité, mémoire de master 1, EHESS, 2006.

18.Dorinda OUTRAM, Georges Cuvier, Vocation, Science and Authority in post-Revolutionnary France, Manchester, Manchester University Press, 1984 ; Michelle SADOUN-GouPIL, Le chimiste Claude-Louis Berthollet. 1748-1822. Sa vie, son œuvre, Paris, Vrin, 1977. 
19. Claude-Isabelle BRELOT, «La noblesse au temps de l'égalité », dans Natalie PeTITEAU (dir.), Voies nouvelles pour l'histoire du Premier Empire, Paris, La Boutique de l'Histoire, 2003, p. 215-224.

20.Lettres d'André Morellet, publiées et annotées par D. MEDLIN et J.C. DAVID, Oxford, The Voltaire Foundation, 1996, t. III. Lettre 472, adressée à Pierre-Louis Roederer, p. 35. 21.Marie-Noëlle BOURGUET, Déchiffrer la France. La Statistique départementale à l'époque napoléonienne, Paris, Éd. des Archives contemporaines, 1989 ; Isabelle LABOULAIS-LESAGE, Lectures et pratiques de l'espace. L'itinéraire de Coquebert de Montbret, savant et grand commis de l'État (1755-1831), Paris, Honoré Champion, 1999.

22.Yves BENOT, La démence coloniale sous Napoléon, préface inédite de Marcel DORIGNY, Paris, La Découverte, 2006, p. 211 et suiv.

23. Claude BLANCKAERT, La nature de la société. Organicisme et sciences sociales au XIX ${ }^{e}$ siècle, Paris, L'Harmattan, 2004.

24.Isabelle LABOULAIS-LESAGE, Lectures et pratiques de l'espace, op. cit., p. 274 : « Le savant est désormais intégré à l'appareil gouvernemental, et il s'appuie pour la réalisation de ses propres projets sur l'aide et les compétences professionnelles des administrateurs ; ce processus conduit au partage des rôles entre celui qui fait avancer la science et celui qui contribue à la diffuser ». Voir aussi Dominique MARGAIRAZ, François de Neufchâteau, op. cit.

25.Jean-Luc CHAPPEY, La Société des Observateurs de l'homme, op. cit., p. 461 et suiv.

26.Jean-Marc-G. ITARD, Rapport fait à son excellence le Ministre de l'Intérieur sur les nouveaux développements et l'état actuel du sauvage de l'Aveyron, Paris, Imprimerie impériale, 1807. 27.François PÉRON, Voyages de découvertes aux terres australes, Paris, Imprimerie nationale, 1807.

28. Marc RENEVILLE, Crime et folie. Deux siècles d'enquêtes médicales et judiciaires, Fayard, 2003, chapitres II et III ; Nicole EDELMAN, Les métamorphoses de l'hystérique. Du début du XIX ${ }^{e}$ siècle à la Grande Guerre, Paris, La Découverte, 2003.

29.Xavier MARTIN, Nature humaine et Révolution française : du siècle des lumières au Code Napoléon, Paris, Dominique Martin Morin, 1994 ; Serge MoussA (dir.), L'idée de " race " dans les sciences humaines et la littérature (XVIII et XIX siècles), Paris, L'Harmattan, 2003. 30.Bruno BELHOSTE, La formation d'une technocratie, op. cit.

31.Dominique GuILLo, « À la recherche des signes de l'identité. Balzac et l'histoire naturelle », Politix, 2006, ${ }^{\circ}$ 74, vol. 19, p. 49-74.

32.Rigomer BAzIN, Les Lettres philosophiques, Paris, chez l'éditeur, rue d'Aboukir, $\mathrm{n}^{\circ} 31$, 1807, p. 539 : «Le mouvement politique qui a bouleversé la France en 1789, n'a changé que les conditions et les fortunes; il a brisé les entraves de la pensée ; mais ce n'est pas assez de rendre sa liberté, il faut encore qu'elle apprenne à s'en servir. La révolution politique est faite; il reste à faire la révolution morale, et elle a besoin, pour s'opérer, d'un Descartes, comme nos états généraux eurent besoin d'un Sieyès, d'un Mirabeau, pour se constituer en législateurs ». Il ajoute plus loin, p. 549 : « Il est donc évident, $1^{\circ}$ Qu'une bonne morale est la base du bonheur des individus et des peuples. $2^{\circ}$ Qu'elle doit résulter de la connaissance de l'homme, sous le rapport de son double instinct de conservation, personnel et collectif. $3^{\circ} \mathrm{Qu}$ 'on pourrait à la rigueur, faire aujourd'hui ce travail, sans un nouveau progrès dans les sciences. $4^{\circ}$ Qu'un nouveau corps de doctrine morale est à faire, dans le $19^{\mathrm{e}}$ siècle, par la philosophie ». Cf. Pierre SERNA, « Rigomer Bazin et la Restauration : penser la République dans la Monarchie ", AHRF, 2001, n³, p. 53-76. 
33.Pietro CORSI, Lamarck. Genèse et enjeux du transformisme 1770-1830, Paris, CNRS Éditions, 2001.

34.En 1812, Moreau de la Sarthe, alors bibliothécaire de la Faculté de médecine de Paris, mène campagne en faveur $\mathrm{du}$ « traitement moral » de Pinel au moment où ce dernier est progressivement marginalisé au sein de l'espace médical : « L'histoire des maladies de l'esprit est une suite ou plutôt un complément nécessaire de l'histoire des passions. Dans un semblable sujet, la médecine sort de l'intérêt local et borné de ses autres études, pour s'unir à la philosophie et à la connaissance pratique du cœur humain, par les plus importantes communications. Par la nature des faits qu'alors elle raconte ou examine, elle ne s'adresse pas seulement aux hommes d'une raison forte et cultivée ; elle a aussi des droits à l'attention des lecteurs moins graves ; elle mérite même peut-être d'occuper ce monde superficiel et aimable des salons, que l'on veut rapprocher aujourd'hui des académies, et qui daigne accueillir la science, quand elle lui est présentée avec des circonstances propres à éveiller sa curiosité, ou à exciter ses émotions [...]. D'après l'importance d'un semblable sujet d'étude, nous avons vu, avec quelque surprise, que l'on eût négligé de faire connaître dans les journaux les plus instructifs, la seconde édition de l'ouvrage de M. Pinel sur les maladies mentales ", Fragments pour servir à l'histoire de la médecine des maladies mentales et de la médecine morale, Paris, 1812, p. 3-4.

35." Nous terminerons cet avertissement en faisant remarquer que les ouvrages de Vicq d'Azyr ne doivent pas tout leur prix à un simple mérite littéraire, ainsi que veulent le faire croire quelques personnes intéressées à une semblable opinion ; qu'ils sont encore recommandables sous le rapport de la science ", dans Euvres de Vicq d'Azyr, éditées par J.L. MOREAU DE LA SARTHE, Paris, Beaudouin, 1805, vol. 1, préface, p. III. 36. À côté de son Histoire naturelle de la femme, Moreau de la Sarthe s'est par exemple intéressé particulièrement aux populations montagnardes « découvertes » par le biais des enquêtes préfectorales : «L'expérience nous a bien appris, sans doute, que l'on ne peut pas simplement chercher à faire arriver d'une manière trop prompte quelques éclairs de raison et de philosophie dans les derniers rangs de la société ; mais ne pourrait-on pas rendre le peuple plus heureux sans l'éclairer trop brusquement ; effacer plus rapidement les traces de barbarie et d'état sauvage qui subsistent encore chez les nations les plus policées ; distribuer ainsi plus uniformément les bienfaits de la civilisation, et faisant arriver moins lentement jusqu'à l'habitant des campagnes tout ce que le progrès des lumières peut apporter de bonheur à son état, remplir un peu l'intervalle qui le sépare de la partie policée et heureuse de la nation ? ", cité par MOREAU DE LA SARTHE, « Considérations sur quelques traces de l'état sauvage chez les peuples policés, et histoire particulière du petit canton de Saterland ", Décade philosophique, 20 fructidor an XII, n 35, p. 457.

37.Il faudrait sans doute s'interroger davantage sur la position de ces acteurs et analyser plus précisément le rôle de certains systèmes de représentations dans la construction de leur identité de savant sous l'Empire (je pense particulièrement à la posture de « l'ermite », qui connaît une nouvelle vogue en cette période).

38. Quelques mémoires sur différents sujets : la plupart d'histoire naturelle ou de physique générale et particulière, Paris, Delance, 1807.

39." Analyse des travaux de la classe des sciences mathématiques et physiques de l'Institut national pendant le second semestre de 1806 ", lu à la séance du 5 janvier 1807, Mémoires de l'Institut National, t. VII, 1806-1807, p. 80-116. 
40.Laurent LоTY, «Métaphysique et science de la nature : Dupont de Nemours contre la théorie de l'instinct ", Nature, Histoire, Société. Essais en hommage à Jacques Roger, Cl. BlANCKAERT, J.L. FIsCHER, R. Rey (dir.), Paris, Klincksieck, 1995, p. 327-340.

41.Pietro CORSI, « Buffon sous la Révolution et l'Empire », dans Jean GAYON (dir.), Buffon 88, Paris, Vrin, 1992, p. 639-648.

42.Journal fondé en 1788 par Parmentier. Il disparait en 1797 et est recréé en 1802.

43.Jean-Luc CHAPPEY, « Enjeux sociaux et politiques de la 'vulgarisation scientifique' en révolution (1780-1810) ", Annales historiques de la Révolution française, 2004, n 338, $\mathrm{p}$. 11-51.

44.Roger MORTIER, « Une revue germanisante sous l'Empire : les Archives littéraires de l'Europe (1804-1808) », Extrait de la Revue de littérature comparée, 1951, p. 43-64.

45.Cité dans Dorinda OutRAM, op. cit., 1984, p. 132.

46.Ibid.

47.Carla HESSE, Publishing and cultural Politics in Revolutionary Paris, 1789-1810, University of California Press, 1991.

48. Une enquête collective sur les secours et pensions distribuées aux savants, hommes de lettres et artistes entre 1770 et 1848 est actuellement mise en place par une équipe de recherche constituée autour de Gilles Malandain, Antoine Lilti, Yasmine Marcil, Barbara Revelli, Pauline Lemaigre, Christelle Rabier, Sophie Wanich, Corinne Legoy et Jean-Luc Chappey.

49.Parmi les nombreux exemples que l'on a trouvés, citons Le Tribunal d'Apollon ou Jugement en dernier ressort de tous les auteurs vivants. Libelle injurieux, partial et diffamatoire par une société de pygmées littéraires [Paris, chez Marchand, an VIII, 2 vol.], rédigé en 1800 par Joseph Rosny en collaboration avec Claude François Félix Mercier de Compiègne et Felix Nogaret et très représentatif de ces productions qui se multiplient sous l'Empire. 50.AN, AF IV 1049. Direction générale de l'imprimerie et de la librairie. $\mathrm{N}^{\circ} 45$, bulletin de la 2nde semaine de novembre 1813.

51.En 1810, un rapport officiel fustige ainsi les membres de la seconde Classe dont « la marche constante et secrète [...] tend sans cesse d'un côté à discréditer le gouvernement et à obscurcir sa gloire et de l'autre à concentrer exclusivement ses bienfaits dans le sein même de l'Institut, et surtout à faire sentir à tout savant, à tout homme de lettres, à tout artiste, que pour arriver aux honneurs littéraires, ce n'est pas au gouvernement, mais à l'institut qu'il faut se dévouer », « Rapport sur les prix décennaux ", AN, AF IV 1050, dossier $n^{\circ} 6$.

52.Annie JOURDAN, Napoléon, héros, imperator, mécène, Paris, Aubier, 1998 ; Catriona SETH, « L'Institut et les prix littéraires", dans Jean.-Claude BonNET (dir.), L'Empire des Muses. Napoléon, les Arts et les Lettres, Paris, Belin, 2004, p. 111-132.

53.C'est le cas des rédacteurs du Spectateur français du XIXe siècle, parmi lesquels on retrouve Chateaubriand, Bonald ou l'abbé de Boulogne. Cf. Jean-Luc CHAPPEY, «Le XVII ${ }^{e}$ siècle comme enjeu philosophique et littéraire au début du XIX siècle », Cahiers $d u$ Centre de Recherches historiques, $n^{\circ}$ 28-29, p. 101-116.

54.Paul BÉNICHOU, Le Sacre de l'écrivain 1750-1830, Paris, Librairie José Corti, 1985. 55.Cette hypothèse doit beaucoup au travail de Corinne LEGOY, Éloges politiques et thuriféraires de la Restauration. Chanter, servir ou combattre, les sens de la célébration, thèse sous la direction d'Alain CORBIN, Université de Paris I, 2004. 
56.Darrin MACMAHON, Enemies of the Enlightenment. The French Conter-Enterlightenment and the making of modernity, Oxford, 2001 ; Jean-Luc CHAPPEY, « Catholiques et sciences au début du XIX ${ }^{\mathrm{e}}$ siècle ", Cahiers d'histoire. Revue d'histoire critique, nº 87, p. 13-36. 57.Je pense particulièrement au grand projet lancé par Henri Grégoire pour recomposer la «République des Lettres » dans son Plan d'association générale entre les savants, gens de lettres et artistes pour accélérer le progrès des bonnes mœurs et des Lumières rédigé entre 1816-1817 Cf. L'abbé Grégoire et la République des Savants, introduction et notes par Bernard Plongeron, Paris, CTHS, 2001.

58.Barbara REVELLI, « L'esprit encyclopédique comme projet politique. La Revue Encyclopédique (1819-1831) », communication inédite au séminaire « Autour des révolutions », ENS Ulm, mai 2006.

59.Annie PETIT, « Le corps scientifique selon Auguste Comte », dans A. KREMER-MARIETTI (dir.), Sociologie de la science, Paris, Mardaga, 1998, p. 69-92.

60.Gérard NoIRIEL, Les fils maudits de la République. L'avenir des intellectuels en France, Paris, Fayard, coll. « Histoire de la pensée », 2005.

61.Claude NICOLET, L'Idée républicaine en France (1789-1924) [1982], Paris, Gallimard, TEL, 1995.

\section{RÉSUMÉS}

Trop souvent présenté comme une période de restauration de l'ordre des lettres et des sciences, l'Empire se caractérise par des transformations profondes touchant autant les modalités épistémologiques et méthodologiques qu'institutionnelles et sociales de construction et de diffusion des savoirs. Face à ce que l'on peut définir comme une "organisation impériale » des savoirs, on voit émerger des résistances nombreuses et hétérogènes dans le monde des lettres et des sciences. Trop souvent ignorées par une historiographie qui tend à les présenter comme anecdotiques ou à les masquer derrière l'intérêt exclusif porté aux groupes d'opposants consacrés (les Idéologues, le groupe de Coppet), ces résistances intellectuelles constituent pourtant des objets d'étude privilégiés pour rendre compte des logiques politiques et des transformations profondes du régime. On tentera en effet de montrer, en précisant les contours de cette organisation impériale des savoirs, comment certaines oppositions dans le domaine des lettres et des sciences ont tenté de défendre l'héritage républicain du Directoire contre les nouvelles formes de normalisation sociale et de domination politique mises en place à partir de 1804. Il s'agira donc de mesurer davantage les rapports particulièrement denses qui unissent les dynamiques intellectuelles et politiques pendant la période.

Republican Heritage and Resistance to the «Organization of Knowledge». Too often presented as a period of restoration of order in the realm of letters and sciences, the Empire is characterized by profound transformations affecting epistemological and methodological structures as much as institutional and social organization and means for the transmission of knowledge. In opposition to what one might call an «imperial organization» of knowledge, one can see emerge numerous and heterogeneous forms of resistances in the world of letters and sciences. Too often ignored by a historiography that tends to present them as anecdotal, and to hide them behind the exclusive interest of official opposing groups (Ideologues, the group of 
Coppet), these forms of intellectual resistance constitute, however, the subject of special study to explain political dynamic and the profound transformation of the regime. I will try to show by specifying the parameters of this imperial organization of knowledge how certain opposition in the realm of letters and sciences attempted to defend the republican heritage of the Directory against new forms of social normalization and political domination established in 1804 . Thus, this study will explore the particularly strong relationships uniting intellectual and political dynamics during the period.

\section{INDEX}

Mots-clés : science de l'homme, Sciences, idéologie, civilisation, progrès

\section{AUTEUR}

\section{JEAN-LUC CHAPPEY}

IHRF, Université de Paris I / Panthéon Sorbonne, 17 rue de la Sorbonne, 75231 Paris cedex 05, jean-luc.chappey@univ-paris1.fr 